\title{
Liver Cancer pMX TNM Finding v6
}

National Cancer Institute

\section{Source}

National Cancer Institute. Liver Cancer pMX TNM Finding v6. NCI Thesaurus. Code C61178.

Liver cancer in which distant metastasis cannot be assessed. (from AJCC 6th Ed.) 